\title{
On the other law of the iterated logarithm for self-normalized sums
}

\section{GUANG-HUI CAI}

Department of Mathematics and Statistics, Zhejiang Gongshang University, Hangzhou 310035, P.R. China

Manuscript received on March 17, 2008; accepted for publication on May 15, 2008;

presented by ARON SIMIS

\section{ABSTRACT}

In this note, we obtain a Chung's integral test for self-normalized sums of i.i.d. random variables. Furthermore, we obtain a convergence rate of Chung law of the iterated logarithm for self-normalized sums.

Key words: Chung's integral test, self-normalized sums, convergence rate.

\section{INTRODUCTION}

Let $X, X_{1}, X_{2}, \ldots$ be i.i.d. random variables with mean zero and variance one, and set

$$
S_{n}=\sum_{k=1}^{n} X_{k}, \quad M_{n}=\max _{1 \leq k \leq n}\left|S_{k}\right| \quad \text { and } \quad V_{n}^{2}=\sum_{k=1}^{n} X_{k}^{2}, \quad n \geq 1 .
$$

Also let $\log x=\ln (x \vee e), \log _{2} x=\log (\log x)$. Then by the so-called Chung's law of the iterated $\operatorname{logarithm}$ we have

$$
\liminf _{n \rightarrow \infty} \sqrt{\log _{2} n / n} M_{n}=\pi / \sqrt{8} \quad \text { a.s. }
$$

This result was first proved by Chung (1948) under $\mathrm{E}|X|^{3}<\infty$, and by Jain and Pruitt (1975) under the sole assumption of a finite second moment. Einmahl (1989) obtained the Darling Erdös theorem for sums of i.i.d. random variables. Griffin and Kuelbs (1989) got Self-normalized laws of the iterated logarithm. Griffin and Kuelbs (1991) obtained some extensions of the laws of the iterated logarithm via self-normalized. Lin (1996) got a self-normalized Chung-type law of iterated logarithm. Einmahl (1993) obtained the following integral test refining (1.1) under the minimal conditions.

THEOREM A. Let $\left\{X, X_{n} ; n \geq 1\right\}$ be a sequence of i.i.d. random variables with $E X=0, E X^{2}=1$ and

$$
E X^{2} I\{|X| \geq t\}=O\left(\left(\log _{2} t\right)^{-1}\right) \text { as } t \rightarrow \infty .
$$

AMS Classification: 60F15, 62F05

E-mail: cghzju@163.com 
Then for any eventually non-decreasing function $\phi:[1, \infty) \rightarrow(0, \infty)$,

$$
\begin{aligned}
& \mathrm{P}\left(M_{n} \leq \sqrt{n} / \phi(n) \text { i.o. }\right)=0 \text { or } 1 \\
& \text { according as } J(\phi):=\int_{1}^{\infty} \frac{\phi(t)^{2}}{t} \exp \left(-\pi^{2} \phi(t)^{2} / 8\right) d t<\infty \text { or }=\infty .
\end{aligned}
$$

Einmahl (1993) showed that if (1.2) is not true, Theorem A is false. We thus see that condition (1.2) is sharp. However, if we use $V_{n}$ to replace $\sqrt{n}$, we can eliminate the condition (1.2) in Theorem A. Explicitly, we get the following theorem.

THEOREM 1.1. Let $\left\{X, X_{n} ; n \geq 1\right\}$ be a sequence of i.i.d. random variables with $E X=0, E X^{2}=1$. Then for any eventually non-decreasing function $\phi:[1, \infty) \rightarrow(0, \infty)$,

$$
\begin{aligned}
& P\left(M_{n} \leq V_{n} / \phi(n) \text { i.o. }\right)=0 \text { or } 1 \\
& \text { according as } J(\phi):=\int_{1}^{\infty} \frac{\phi(t)^{2}}{t} \exp \left(-\pi^{2} \phi(t)^{2} / 8\right) d t<\infty \text { or }=\infty .
\end{aligned}
$$

Our next theorem gives a result on a convergence rate of (1.1).

THEOREM 1.2. Let $\left\{X, X_{n} ; n \geq 1\right\}$ be a sequence of i.i.d. random variables with $E X=0, E X^{2}=1$. Then for any $b>-1$, we have

$$
\sum_{n=1}^{\infty} \frac{\left(\log _{2} n\right)^{b}}{n \log n} \mathrm{P}\left(M_{n} \leq \varepsilon \sqrt{\pi^{2} V_{n}^{2} /\left(8 \log _{2} n\right)}\right)<\infty, \quad \forall \varepsilon>0 .
$$

Throughout this note, let $C$ denote a positive constant, whose values can differ in different places.

\section{PROOF}

PROOF OF THEOREM 1.1. It is enough to prove the result for eventually non-decreasing function $\phi$ : $[1, \infty) \rightarrow(0, \infty)$ satisfying

$$
\frac{1}{2}\left(\log _{2} t\right)^{1 / 2} \leq \phi(t) \leq\left(\log _{2} t\right)^{1 / 2}, \quad t \geq 1
$$

(See Einmahl 1993). Let

$$
\begin{gathered}
X_{1 j}=X_{j} I\left\{\left|X_{j}\right| \leq \sqrt{j} /\left(\log _{2} j\right)^{2}\right\}, \quad j \geq 1 \\
B_{n}^{2}=\sum_{i=1}^{n} \mathrm{E} X_{1 i}^{2} \text { and } \triangle_{n}=\left|\frac{M_{n}}{B_{n}}-\frac{M_{n}}{V_{n}}\right|, \quad n \geq 1 .
\end{gathered}
$$

Observe that by $(2.1)$

$$
\begin{aligned}
& \mathrm{P}\left(M_{n} \leq V_{n} / \phi(n) \text { i.o. }\right) \\
\leq & \mathrm{P}\left(M_{n} \leq V_{n} / \phi(n), \triangle_{n} \geq\left(\log _{2} n\right)^{-3 / 2} \text { i.o. }\right)+\mathrm{P}\left(M_{n} \leq V_{n} / \phi(n), \triangle_{n} \leq\left(\log _{2} n\right)^{-3 / 2} \text { i.o. }\right) \\
\leq & \mathrm{P}\left(M_{n} \leq V_{n} / \phi(n), \triangle_{n} \geq\left(\log _{2} n\right)^{-3 / 2} \text { i.o. }\right)+\mathrm{P}\left(M_{n} / B_{n} \leq \phi(n)^{-1}+\left(\log _{2} n\right)^{-3 / 2} \text { i.o. }\right) \\
\leq & \mathrm{P}\left(M_{n} \leq V_{n} / \phi(n), \triangle_{n} \geq\left(\log _{2} n\right)^{-3 / 2} \text { i.o. }\right)+\mathrm{P}\left(M_{n} \leq B_{n} / \Psi(n) \text { i.o. }\right),
\end{aligned}
$$


where $\Psi(t)=\phi(t)^{3} /\left(1+\phi(t)^{2}\right), t \geq 1$, and similarly,

$$
\begin{aligned}
& \mathrm{P}\left(M_{n} \leq V_{n} / \phi(n) \text { i.o. }\right) \\
\geq & \mathrm{P}\left(M_{n} / B_{n} \leq \phi(n)^{-1}-\left(\log _{2} n\right)^{-3 / 2} \quad \text { i.o. }\right)-\mathrm{P}\left(M_{n} \leq B_{n} / \phi(n), \triangle_{n} \geq\left(\log _{2} n\right)^{-3 / 2} \text { i.o. }\right) \\
\geq & \mathrm{P}\left(M_{n} \leq B_{n} / \Psi^{\prime}(n) \text { i.o. }\right)-\mathrm{P}\left(M_{n} \leq B_{n} / \phi(n), \triangle_{n} \geq\left(\log _{2} n\right)^{-3 / 2} \text { i.o. }\right),
\end{aligned}
$$

where $\Psi^{\prime}(t)=\phi(t)^{3} /\left(\phi(t)^{2}-1\right), t \geq 1$. It is easily checked that $J(\phi)<\infty$ implies $J(\Psi)<\infty$ and $J(\phi)=\infty$ implies $J\left(\Psi^{\prime}\right)=\infty$, and by Theorem 1 of Einmahl (1993), we have

$$
J(\Psi)<\infty \Longrightarrow \mathrm{P}\left(M_{n} \leq B_{n} / \Psi(n) \text { i.o. }\right)=0
$$

and

$$
J\left(\Psi^{\prime}\right)=\infty \Longrightarrow \mathrm{P}\left(M_{n} \leq B_{n} / \Psi^{\prime}(n) \text { i.o. }\right)=1 .
$$

Now by Lemma 2.2 below,

$$
\mathrm{P}\left(M_{n} \leq V_{n} / \phi(n), \Delta_{n} \geq\left(\log _{2} n\right)^{-3 / 2} \text { i.o. }\right)=0
$$

and

$$
\mathrm{P}\left(M_{n} \leq B_{n} / \phi(n), \triangle_{n} \geq\left(\log _{2} n\right)^{-3 / 2} \text { i.o. }\right)=0 .
$$

From these equations and (2.2), (2.3), hence we see that Theorem 1.1 holds true.

We now present two lemmas used in the main proof of Theorem 1.1.

Lemma 2.1. For any $x>0$ there exist positive constants $\eta=\eta(x)$ and $A=A(x)$ such that

$$
\mathrm{P}\left(M_{n} \leq x \sqrt{n / \log _{2} n}\right) \leq A(\log n)^{-\eta} .
$$

Proof. See the Lemma 2(b) of Einmahl (1993).

LEMMA 2.2. We have

$$
\mathrm{P}\left(M_{n} \leq V_{n} / \phi(n), \triangle_{n} \geq\left(\log _{2} n\right)^{-3 / 2} \text { i.o. }\right)=0
$$

and

$$
\mathrm{P}\left(M_{n} \leq B_{n} / \phi(n), \triangle_{n} \geq\left(\log _{2} n\right)^{-3 / 2} \text { i.o. }\right)=0 .
$$

ProOF. Let

$$
X_{2 j}=X_{j} I\left\{\sqrt{j} /\left(\log _{2} j\right)^{2}<\left|X_{j}\right| \leq \sqrt{j}\right\}, X_{3 j}=X_{j}-X_{1 j}-X_{2 j}, \quad j \geq 1
$$

and

$$
V_{1 n}=\sum_{k=1}^{n}\left(X_{1 k}^{2}-\mathrm{E} X_{1 k}^{2}\right), V_{2 n}=\sum_{k=1}^{n} X_{3 k}^{2}, \quad n \geq 1
$$


First using $\mathrm{E} X^{2}=1$, we have

$$
\begin{aligned}
& \sum_{n=1}^{\infty} \frac{\left(\log _{2} n\right)^{4}}{n^{2}} \mathrm{E}\left(X_{1 n}^{2}-\mathrm{E} X_{1 n}^{2}\right)^{2} \leq 2 \sum_{n=1}^{\infty} \frac{\left(\log _{2} n\right)^{4}}{n^{2}} \mathrm{E} X^{4} I\left\{|X| \leq \sqrt{n} /\left(\log _{2} n\right)^{2}\right\} \\
& \quad=2 \sum_{k=1}^{\infty} \mathrm{E} X^{4} I\left\{\sqrt{k-1} /\left(\log _{2}(k-1)\right)^{2}<|X| \leq \sqrt{k} /\left(\log _{2} k\right)^{2}\right\} \sum_{n=k}^{\infty} \frac{\left(\log _{2} n\right)^{4}}{n^{2}} \\
& \quad \leq C \sum_{k=1}^{\infty} \mathrm{E} X^{2} I\left\{\sqrt{k-1} /\left(\log _{2}(k-1)\right)^{2}<|X| \leq \sqrt{k} /\left(\log _{2} k\right)^{2}\right\} \\
& \quad \leq C \mathrm{E} X^{2}<\infty
\end{aligned}
$$

and

$$
\sum_{n=1}^{\infty} \mathrm{P}\left(X_{3 n} \neq 0\right)=\sum_{n=1}^{\infty} \mathrm{P}(|X|>\sqrt{n}) \leq C \mathrm{E} X^{2}<\infty
$$

Thus, it follows by applying Corollary 3.1 of Lin et al. (1999, P.95) and Borel-Cantelli lemma that

$$
\frac{\left(\log _{2} n\right)^{2}}{n} V_{1 n} \rightarrow 0 \text { a.s. and } V_{2 n}=O(1) \text { a.s. }
$$

Using strong law of large numbers and Hartman-Wintner LIL, we have

$$
\lim _{n \rightarrow \infty} \frac{V_{n}^{2}}{n}=1 \quad \text { a.s. } \text { and } \limsup _{n \rightarrow \infty} \frac{M_{n}}{\sqrt{2 n \log _{2} n}} \leq 1 \text { a.s. }
$$

Thus, by $\mathrm{E} X^{2}=1$, we obtain that for large $n$,

$$
\begin{aligned}
\triangle_{n} & =\left|\frac{M_{n}\left(V_{n}^{2}-B_{n}^{2}\right)}{B_{n} V_{n}\left(B_{n}+V_{n}\right)}\right| \\
& \leq \frac{3 \sqrt{\log _{2} n}}{n}\left(\left|V_{1 n}\right|+V_{2 n}+\sum_{j=1}^{n} X_{2 j}^{2}\right) \\
& \leq \frac{3 \sqrt{\log _{2} n}}{n}\left(\left|V_{1 n}\right|+V_{2 n}+\sqrt{n} \sum_{j=1}^{n}\left|X_{2 j}\right|\right) \text { a.s. }
\end{aligned}
$$

Recalling that $B_{n}^{2} \leq n, n \geq 1$ and $\lim _{n \rightarrow \infty} V_{n}^{2} / n=1$ a.s., in order to prove (2.4) and (2.5), by (2.1), (2.6) and (2.7), it suffices to show that

$$
\mathrm{P}\left(M_{n} \leq 2 \sqrt{n / \log _{2} n}, \sum_{j=1}^{n}\left|X_{2 j}\right| \geq \frac{1}{4} \sqrt{n} /\left(\log _{2} n\right)^{2} \text { i.o. }\right)=0 .
$$

Now, set $m(n):=\left[n /\left(\log _{2} n\right)^{9}\right], n \geq 1$. By $\mathrm{E} X^{2}=1$, we have

$$
\sum_{j=1}^{n} \mathrm{E}\left|X_{2 j}\right| \leq \sum_{j=1}^{n} \mathrm{E}\left|X_{j}\right| I\left\{\left|X_{j}>\sqrt{j} /\left(\log _{2} j\right)^{2}\right|\right\} \leq \sum_{j=1}^{n} \frac{\left(\log _{2} j\right)^{2}}{\sqrt{j}} \leq C \sqrt{n}\left(\log _{2} n\right)^{2} .
$$


Applying Kolmogorov's LIL and $\mathrm{E} X^{2}=1$, we have

$$
\limsup _{n \rightarrow \infty} \frac{\sum_{j=1}^{n}\left(\left|X_{2 j}\right|-\mathrm{E}\left|X_{2 j}\right|\right)}{\sqrt{2 n \log _{2} n}} \leq 2 \text { a.s. }
$$

it easily follows from above inequalities that

$$
\sum_{j=1}^{m(n)}\left|X_{2 j}\right|=o\left(\sqrt{n} /\left(\log _{2} n\right)^{2}\right) \quad \text { a.s. }
$$

Hence observe that on account of (2.9) it is enough to show that

$$
\mathrm{P}\left(M_{n} \leq 2 \sqrt{n / \log _{2} n}, \sum_{j=m(n)+1}^{n}\left|X_{2 j}\right| \geq \frac{1}{5} \sqrt{n} /\left(\log _{2} n\right)^{2} \text { i.o. }\right)=0 .
$$

Let $n_{k}=2^{k}$ and $m_{k}=\left[2^{k} /(\log k)^{10}\right], k \geq 0$, for large enough $k$,

$$
\begin{aligned}
& \bigcup_{n=n_{k-1}+1}^{n_{k}}\left\{M_{n} \leq 2 \sqrt{n / \log _{2} n}, \sum_{j=m(n)+1}^{n}\left|X_{2 j}\right| \geq \frac{1}{5} \sqrt{n} /\left(\log _{2} n\right)^{2}\right\} \\
& \subseteq\left\{M_{n_{k-1}} \leq 2 \sqrt{2 n_{k-1} / \log _{2} n_{k-1}}, \sum_{j=m_{k}+1}^{n_{k}}\left|X_{2 j}\right| \geq \frac{1}{10} \sqrt{n_{k}} /\left(\log _{2} n_{k}\right)^{2}\right\} .
\end{aligned}
$$

Thus, in order to prove (2.10), it suffices to show that

$$
\mathrm{P}\left(M_{n_{k-1}} \leq 2 \sqrt{2 n_{k-1} / \log _{2} n_{k-1}}, \sum_{j=m_{k}+1}^{n_{k}}\left|X_{2 j}\right| \geq \frac{1}{10} \sqrt{n_{k}} /\left(\log _{2} n_{k}\right)^{2} \text { i.o. }\right)=0 .
$$

Let

$$
M_{n_{k-1}, j}=M_{j-1} \vee \max _{j<n \leq n_{k-1}}\left|S_{n}-X_{j}\right|, \quad 1 \leq j \leq n_{k-1} \quad \text { and } \quad n_{k}^{\prime}=n_{k-1}-1 .
$$

Notice that

$$
M_{n_{k-1}, j} \leq M_{n_{k-1}}+\left|X_{j}\right| \leq 3 M_{n_{k-1}}, \quad 1 \leq j \leq n_{k-1} .
$$

Using the independence and Lemma 2.1, it is clear that for some constant $\eta>0$ and large enough $k$,

$$
\begin{aligned}
& \mathrm{P}\left(M_{n_{k-1}} \leq 2 \sqrt{2 n_{k-1} / \log _{2} n_{k-1}}, \sum_{j=m_{k}+1}^{n_{k}}\left|X_{2 j}\right| \geq \frac{1}{10} \sqrt{n_{k}} /\left(\log _{2} n_{k}\right)^{2}\right) \\
\leq & \mathrm{P}\left(\bigcup_{j=m_{k}+1}^{n_{k}}\left\{\left|X_{j}\right|>\sqrt{j} /\left(\log _{2} j\right)^{2}, M_{n_{k-1}} \leq 2 \sqrt{2 n_{k-1} / \log _{2} n_{k-1}}\right\}\right) \\
\leq & \sum_{j=m_{k}+1}^{n_{k-1}} \mathrm{P}\left(\left|X_{j}\right|>\sqrt{j} /\left(\log _{2} j\right)^{2}, M_{n_{k-1}} \leq 2 \sqrt{2 n_{k-1} / \log _{2} n_{k-1}}\right) \\
& +\sum_{j=n_{k-1}+1}^{n_{k}} \mathrm{P}\left(\left|X_{j}\right|>\sqrt{j} /\left(\log _{2} j\right)^{2}, M_{n_{k-1}} \leq 2 \sqrt{2 n_{k-1} / \log _{2} n_{k-1}}\right)
\end{aligned}
$$




$$
\begin{aligned}
\leq & \sum_{j=m_{k}+1}^{n_{k-1}} \mathrm{P}\left(\left|X_{j}\right|>\sqrt{j} /\left(\log _{2} j\right)^{2}, M_{n_{k-1}, j} \leq 6 \sqrt{2 n_{k-1} / \log _{2} n_{k-1}}\right) \\
& +\sum_{j=n_{k-1}+1}^{n_{k}} \mathrm{P}\left(\left|X_{j}\right|>\sqrt{j} /\left(\log _{2} j\right)^{2}\right) \mathrm{P}\left(M_{n_{k-1}} \leq 2 \sqrt{2 n_{k-1} / \log _{2} n_{k-1}}\right) \\
\leq & \sum_{j=m_{k}+1}^{n_{k-1}} \mathrm{P}\left(\left|X_{j}\right|>\sqrt{j} /\left(\log _{2} j\right)^{2}\right) \mathrm{P}\left(M_{n_{k}^{\prime}} \leq 9 \sqrt{n_{k}^{\prime} / \log _{2} n_{k}^{\prime}}\right) \\
& +\sum_{j=n_{k-1}+1}^{n_{k}} \mathrm{P}\left(\left|X_{j}\right|>\sqrt{j} /\left(\log _{2} j\right)^{2}\right) \mathrm{P}\left(M_{n_{k-1}} \leq 3 \sqrt{n_{k-1} / \log _{2} n_{k-1}}\right) \\
\leq & C k^{-\eta} \sum_{j=m_{k}+1}^{n_{k}} \mathrm{P}\left(|X|>\sqrt{j} /\left(\log _{2} j\right)^{2}\right) .
\end{aligned}
$$

Finally, By Lemma 4 of Einmahl (1993), we have

$$
\begin{aligned}
& \sum_{k=1}^{\infty} \mathrm{P}\left(M_{n_{k-1}} \leq 2 \sqrt{2 n_{k-1} / \log _{2} n_{k-1}}, \sum_{j=m_{k}+1}^{n_{k}}\left|X_{2 j}\right| \geq \frac{1}{10} \sqrt{n_{k}} /\left(\log _{2} n_{k}\right)^{2}\right) \\
\leq & C \sum_{k=1}^{\infty} k^{-\eta} \sum_{j=m_{k}+1}^{n_{k}} \mathrm{P}\left(|X|>\sqrt{j} /\left(\log _{2} j\right)^{2}\right)<\infty
\end{aligned}
$$

and hence we obtain (2.11) from the Borel-Cantelli lemma.

Proof OF TheOREM 1.2. For each $n \geq 1$ and $1 \leq i \leq n$, we have

$$
\bar{X}_{n i}=X_{i} I\left\{\left|X_{i}\right| \leq n^{1 / 2}(\log n)^{-1 / 3}\right\}, \quad \bar{V}_{n}^{2}=\sum_{j=1}^{n} \bar{X}_{n j}^{2} \quad \text { and } \quad \bar{B}_{n}^{2}=\sum_{j=1}^{n} \operatorname{Var}\left(\bar{X}_{n j}\right) .
$$

By $\mathrm{E} X^{2}=1$, it is easy to show that $\bar{B}_{n}^{2} \leq n$. Hence for some $\frac{1}{7}<\delta<1$ and any $\varepsilon>0$

$$
\begin{aligned}
& \mathrm{P}\left(M_{n} \leq \varepsilon \sqrt{\pi^{2} V_{n}^{2} /\left(8 \log _{2} n\right)}\right) \\
\leq & \mathrm{P}\left(M_{n} \leq \varepsilon \sqrt{\pi^{2}(1+\delta) \bar{B}_{n}^{2} /\left(8 \log _{2} n\right)}\right)+\mathrm{P}\left(V_{n}^{2} \geq(1+\delta) \bar{B}_{n}^{2}\right) \\
\leq & \mathrm{P}\left(M_{n} \leq \varepsilon \sqrt{\pi^{2}(1+\delta) / 8} \sqrt{n / \log _{2} n}\right)+\mathrm{P}\left(\bar{V}_{n}^{2} \geq(1+\delta) \bar{B}_{n}^{2}\right) \\
& +\mathrm{P}\left(\bigcup_{j=1}^{n}\left\{\left|X_{j}\right|>n^{1 / 2}(\log n)^{-1 / 3}\right\}\right) \\
& =I_{1}+I_{2}+I_{3} .
\end{aligned}
$$

In order to prove (1.5), it suffices to show that for any $b>-1$

$$
\sum_{n=1}^{\infty} \frac{\left(\log _{2} n\right)^{b}}{n \log n} I_{i}<\infty, \quad \forall \varepsilon>0, \quad i=1,2,3
$$


By Lemma 2.1, there exists a positive constant $\eta$ such that

$$
\sum_{n=1}^{\infty} \frac{\left(\log _{2} n\right)^{b}}{n \log n} I_{1} \leq C \sum_{n=1}^{\infty} \frac{\left(\log _{2} n\right)^{b}}{n \log n}(\log n)^{-\eta}<\infty, \quad \forall \varepsilon>0 .
$$

Since $\mathrm{E} X=0$ and $\mathrm{E} X^{2}=1$, there exists a positive integer $n_{0}$ such that for all $n \geq n_{0}$

$$
\mathrm{E} \bar{X}_{n 1}^{2} \geq \frac{3}{4} \text { and } \mathrm{E} \bar{X}_{n 1} \leq \frac{1}{4}
$$

Hence using the Bernstein inequality, there exists a positive constant $\beta<1 / 3000$ such that

$$
\begin{aligned}
\sum_{n=1}^{\infty} \frac{\left(\log _{2} n\right)^{b}}{n \log n} I_{2} & \leq C+\sum_{n=n_{0}}^{\infty} \frac{\left(\log _{2} n\right)^{b}}{n \log n} \mathrm{P}\left(\bar{V}_{n}^{2} \geq(1+\delta / 2) n \mathrm{E} \bar{X}_{n 1}^{2}\right) \\
& \leq C+\sum_{n=n_{0}}^{\infty} \frac{\left(\log _{2} n\right)^{b}}{n \log n}(\log n)^{-\beta} \\
& <\infty
\end{aligned}
$$

Finally, by $\mathrm{E} X^{2}=1$, we have

$$
\sum_{n=1}^{\infty} \frac{\left(\log _{2} n\right)^{b}}{n \log n} I_{3} \leq \sum_{n=1}^{\infty} \frac{\left(\log _{2} n\right)^{b}}{\log n} \mathrm{P}\left(|X|>n^{1 / 2}(\log n)^{-1 / 3}\right) \leq C \mathrm{E} X^{2}<\infty
$$

Thus, (2.12) holds true.

\section{ACKNOWLEDGMENTS}

This paper was supported by National Natural Science Foundation of China and Youth Talent Foundation of Zhejiang Gongshang University (Q07-07).

\section{RESUMO}

Nesta nota, obtemos um teste integral de Chung para somas auto-normalizadas de variáveis aleatórias i.i.d. (independentes e identicamente distribuídas). Além disso, obtemos uma taxa de convergência da lei de Chung do logaritmo iterado para somas auto-normalizadas.

Palavras-chave: teste integral de Chung, somas auto-normalizadas, taxa de convergência.

\section{REFERENCES}

CHUNG KL. 1948. On the maximal partial sums of sequences of independent random variables. Trans Amer Math Soc 4: 205-233.

Einmahl U. 1989. The Darling Erdös theorem for sums of i.i.d. random variables. Probab Theory Relat Fields 82: $241-257$.

EinMahl U. 1993. On the other law of the iterated logarithm. Probab Theory Relat Fields 96: 97-106. 
GRIFFIN PS AND KUELBS JD. 1989. Self-normalized laws of the iterated logarithm. Ann Probab 17: 1571-1601.

GRIFFIN PS AND KUELBS JD. 1991. Some extensions of the laws of the iterated logarithm via self-normalized. Ann Probab 19: 380-395.

JAIN NC And PRUitT WE. 1975. The other law of the iterated logarithm. Ann Probab 3: 1046-1049.

LIN ZY. 1996. A self-normalized Chung-type law of iterated logarithm. Theory Probab Appl 41: 791-798.

LIN ZY, LU CR AND SU AG. 1999. Limit Theoretical Basis of Probability. Hight Education Press, Beijing. 